\title{
Poster Highlights for SPS4
}

\section{Research, Curriculum, and Resources}

Measuring the Circumference of the Earth in Primary School. Emmanuel DiFolco (France). The French program "La Main à la Pate" is leading an international co-operative project, gathering each year more than 100 schools all over the world. The project invites teachers and pupils in primary schools to measure the circumference of the Earth, following the method first developed by the Greek scientist Eratosthenes, 2200 years ago.

The protocol consists of a series of experimental activities which allow a progressive approach to the various scientific notions at play (light rays and shadows, the shape of the Earth, solar noon etc.) Pupils are invited to reproduce the observations of Eratosthenes, and to adapt the method by developing their own instruments. Finally, they can compute their own estimate of the size of our planet by exchanging their measurements easily through the Internet with other classes from many countries.

We present the progressive protocol and its entertaining activities, as well as the specific cooperative tools which have been created to help and follow up the teachers in the course of the project: a scientists' and trainers' network, an Internet forum, and a database where all the measurements gathered can be exchanged between the participants.

Using Games to Teach Astronomy. Paul J. Francis (Australia). We all know that astronomical research is a chaotic, sociable, deeply human enterprise, full of baffling mysteries, enigmatic clues, and breathtakingly unexpected conclusions. Abundant evidence suggests that our students see astronomy very differently. They see it as a lonely activity: a collection of facts (and very pretty pictures) brought down from the mountain by antisocial "experts" for them to memorize.

Can we change this false perception? I've been experimenting with using role-playing games in the classroom. I've tried these games out on a wide range of high school and university students. Students play the roles of competing teams of astronomers, battling to solve some perplexing astrophysical enigma.

Do these games work? Sometimes! When they work well, they really change perceptions of science, in a way that almost no other teaching technique can match. But there have been a fair number of embarrassing fiascos along the way. I will share my experiences, and hard-earned tips for avoiding disasters.

Mission to Mars (MTM) at Bob Jones University. R. Samec (USA). MTM develops interest in space exploration through a highly realistic, simulated trip to Mars. Students study and learn to appreciate the challenges of space travel including propulsion, life support, medicine, planetary astronomy, psychology, robotics, and communication. Broken into teams (Management, Spacecraft Design, Communications, Life Support, Navigation, Robotics, and Science), they address the problems specific to each aspect of the mission. Teams also learn to interact and recognize that coordinated cooperation is needed for a successful mission. 
Critical and Scientific Thinking in Astronomy Courses. Harry Shipman (USA). This paper summarizes the results of several studies, many conducted with science education collaborators (Nancy W. Brickhouse, Zoubeida Dagher, and Will Letts). We have found that students can and do learn to appreciate evidence, and many learn how to cite evidence to support scientific claims. While students can learn the relationship between evidence and core scientific theories like the Big Bang Theory, their understanding of this relationship depends on the theory, in contrast to previous assertions in the science education literature. Students show particular difficulties in understanding the evidence in support of stellar evolution. Student understanding of the nature of scientific theories is more problematic. However, teaching sequences constructed as a result of this research shows some promise. This paper contains specific examples of teaching strategies used to help students learn critical thinking and scientific habits of mind.

\section{Teacher Education}

Astronomical Network for Teachers in Thailand. B. Hutawarakorn, B. Soonthornthum, S. Poshyachinda, A. Sooksawas (Thailand). We report the latest development of a pilot project in establishing the astronomical network for teachers in Thailand. The project is granted by the Institute for the Promotion of Teaching Science and Technology, Thailand and operated by Sirindhorn Observatory, Chiangmai University. The objectives of the project are to establish semi-robotic telescopes which can be accessed from schools nationwide, and to establish an educational website in Thai language (http://www.astroschool.in.th) which contains education resources and links to other educational websites worldwide. The network will play an important role in the development of teaching and learning astronomy in Thailand.

Astronomy Education in a Primary Teacher Training Institute. W.R. McIntyre (New Zealand). Teacher self-efficacy for pre-service teachers is dependent on subject-matter knowledge and pedagogical content knowledge (PCK). Students who took a paper (course) entitled Spaceship Earth and Beyond, in addition to the compulsory science methods paper showed a statistically positive correlation between their belief that they could teach astronomical concepts effectively, and their own astronomy understanding. The result is due mainly to the fact that Spaceship Earth and Beyond specifically addresses the subject matter knowledge necessary to teach the astronomy in the science curriculum, and the PCK through the use of the "investigating with models" approach.

What Should We Teach? Goals for Astronomy Courses for Teachers. Bruce Partridge and George Greenstein (USA). We present a list of broad goals for university survey courses designed for non-specialists, including future teachers. Consensus on these goals was reached by leading astronomers and education experts in the US.

These broad goals are specifically not a prescribed curriculum for university astronomy courses. We suggest, however, that university courses meeting these goals would provide excellent training in the methods and values of physical 
science for secondary school teachers. They may also provide useful guidance in the construction of school curricula which have some astronomy content.

These meetings were sponsored by the American Astronomical Society, and supported, in part, by the US National Science Foundation. The full report can be found at:

$$
\text { www.aas.org/education/aasprojects/101-FinalReport.pdf }
$$

\section{Remote/Robotic Telescopes}

The Faulkes Telescope Project. P. O'Brien (UK). The Faulkes Telescope Project is constructing two $2 \mathrm{~m}$ robotic telescopes to be located in Hawaii and Australia. Available from 2004, these will be the world's most powerful telescopes dedicated to education. The University of Leicester, and Liverpool John Moores University will provide optical spectrographs for the telescopes to encourage school usage of this unique facility. To illustrate the telescopes' research-level capability, we will also use the spectrographs to study gamma-ray bursts, the most powerful explosive events in the universe. These will be identified by the NASA Swift satellite, due for launch in early 2004 . These data will also be made available to schools, thereby raising the profile of physics and astronomy in the educational community.

\section{http://www.faulkes-telescope.com}

Observing across Continents. Nick Lomb (Australia), Dan Klinglesmith III and Jim Kotoski (USA). Real-time use of remote telescopes can bring the excitement of professional observing into the classroom. By linking with remote telescopes across time zones and continents, it is possible to carry out observations during normal school hours. We have been collaborating to provide real-time telescope observing to students on both continents.

We have found that real-time observing is an exciting experience for the students, and it gives them ownership of their observations. The presence of an observer at the telescope in text communication with the students greatly enhanced their experience.

Teaching Astronomy and Telescope Use in a High School. Yukimasa Tsubota, Naoki Matsumoto, Minoru Omote and Takeshi Satoh (Japan). Our recommendations are as follows: (a) Small telescopes should be introduced in a high school considering the national curriculum of Japan; (b) Internet Astronomical Observatory (iAO) should be utilized after direct experience with astronomical observation. It turned out through our activities that iAO should have a wide-field sky camera in order to show the movement of the constellations. These may be fixed ones that are directed to different direction such as east, south, west, and north. The real motion of the stars is slow so the snapshot at every hour can be stored and viewed though the Internet. 